ISSN 0103-9954

\title{
ANÁLISE COMPARATIVA DAS PROPRIEDADES FÍSICAS E MECÂNICAS DA MADEIRA DE TRÊS CONÍFERAS DE FLORESTAS PLANTADAS
}

\section{COMPARATIVE ANALYSIS OF PHYSICAL AND MECHANICAL PROPERTIES OF WOOD FROM THREE PLANTATION SOFTWOODS}

\author{
Elio José Santini ${ }^{1}$ Clóvis Roberto Haselein ${ }^{2}$ Darci Alberto Gatto ${ }^{3}$
}

\section{RESUMO}

Madeiras provenientes de florestas plantadas de Araucaria angustifolia, Pinus elliottii e Pinus taeda submetidas a, pelo menos, um desbaste, foram testadas com o objetivo de comparar suas propriedades físicas e mecânicas. Cinco árvores de cada espécie, obtidas de talhões, previamente, selecionados, foram coletadas, transformadas em toretes e pranchões para posterior confecção, condicionamento e ensaio dos corpos de prova segundo as recomendações da norma ASTM. Embora a resistência média da Araucaria tenha sido ligeiramente superior às duas espécies de Pinus, diferenças significativas foram encontradas apenas nos testes de flexão estática (módulo de elasticidade e tensão no limite proporcional), compressão axial (tensão no limite proporcional, módulo de ruptura e de elasticidade) e dureza axial. Entre as espécies de Pinus, não foram dectadas diferenças nas propriedades, exceto em tração normal às fibras em que a resistência da madeira de $P$. taeda foi maior que as demais. Com relação à massa específica básica e coeficiente de contração, nenhuma diferença significante foi observada entre as espécies estudadas.

Palavras-chave: Propriedades, araucária, Pinus elliottii, Pinus taeda.

\begin{abstract}
Woods from plantation stands of parana, slash and loblolly pines, thinned at least once, were tested with the objective of comparing physical and mechanical properties. Five threes from each specie were harvested from selected stands. The stems were bucked into logs and then transported to the sawmill cooperating in this study. The logs were processed into planks, which were air seasoned, and then cut into pieces according to ASTM standards. Although the average strength of parana pine was slightly larger than the two other pines, significant differences were found only in the tests of static bending (modulus of elasticity and tension at the proportional limit), compression parallel to the grain (maximum crushing strength, modulus of elasticity and tension at the proportional limit) and hardness (cross-section). The properties were not different for slash and

1. Engenheiro Florestal, Dr., Professor Adjunto do Departamento de Ciências Florestais, Centro de Ciências Rurais, Universidade Federal de Santa Maria, CEP 97105-900, Santa Maria (RS).

2. Engenheiro Florestal, PhD., Professor Adjunto do Departamento de Ciências Florestais, Centro de Ciências Rurais, Universidade Federal de Santa Maria, CEP 97105-900, Santa Maria (RS).

3. Engenheiro Florestal, Mestrando do Programa de Pós-Graduação em Engenharia Florestal, Centro de Ciências Rurais, Universidade Federal de Santa Maria, CEP 97105-900, Santa Maria (RS).
\end{abstract}


loblolly pines, except in tension perpendicular to the grain, where the strength of loblolly pine was higher than the others. No differences were found for specific gravity and shrinkage among the species.

Key words: Wood properties, parana pine, slash pine, loblolly pine.

\section{INTRODUÇÃO}

A Araucaria angustifolia foi considerada durante um longo período a principal matéria-prima madeireira de importância econômica no sul do País. A exploração desordenada, realizada desde o início do século, reduziu drasticamente suas reservas, trazendo dificuldades para a indústria madeireira que não se preveniu contra a falta do produto.

Nas décadas de 60 e 70, mediante incentivos governamentais, foram introduzidas espécies exóticas de rápido crescimento, com o objetivo de aumentar os estoques de madeira para abastecer a indústria. No sul do País, as coníferas que melhor se adaptaram foram Pinus elliottii e Pinus taeda, originários do sul dos Estados Unidos, bem como espécies do gênero Eucalyptus, uma folhosa procedente da Austrália. Todavia, os projetos deveriam contemplar, por exigência legal, uma determinada porcentagem da área com espécies nativas, e a araucária foi uma das escolhidas.

Atualmente, a disponibilização da matéria-prima, proveniente de florestas plantadas dessas coníferas, induz o mercado consumidor a preocupar-se com a qualidade da madeira. O conhecimento de suas propriedades fornece informações importantes para as diversas fases de processamento industrial, bem como para a utilização do produto final. Entretanto, as características tecnológicas da madeira variam com a espécie, procedência, idade, sítio e altitude (LADRACH, 1986). O efeito da localização geográfica sobre o módulo de ruptura (MOR) e módulo de elasticidade (MOE) em flexão estática foi comprovado por McALISTER \& CLARK III (1991). Além disso, particularmente nos gêneros Pinus e Araucaria, as características tecnológicas são influenciadas também pela presença de madeira com propriedades diferenciadas, como lenhos de reação, juvenil, inicial e tardio (KELLISON, 1981).

O lenho juvenil é indesejável por ser menos resistente e mais flexível do que o lenho adulto, em razão da menor massa específica, traqueóides mais curtos, maiores ângulos fibrilares, paredes celulares mais finas, maiores diâmetros de lumens, menor percentagem de lenho tardio, mais lenho de compressão e alta contração longitudinal (BIBLIS et al., 1993; PEARSON \& GILMORE, 1971).

Em geral, a madeira de árvores mais jovens, constituída de alta percentagem de lenho juvenil na seção do tronco, possui propriedades mecânicas mais baixas que nas árvores maduras. PEARSON \& GILMORE (1980) demonstraram que o MOR em madeiras juvenil e adulta de árvores de quinze anos correspondeu, em média, a 48 e 76\%, respectivamente, do lenho adulto de árvores com 41 anos de idade.

O WOOD HANDBOOK (1987) relata que a madeira de $P$. elliottii e $P$. taeda, respectivamente, possue massa específica básica de 0,54 e $0,47 \mathrm{~g} / \mathrm{cm}^{3}$, e MOE de 138000 e 125000 $\mathrm{kgf} / \mathrm{cm}^{2}$ a $12 \%$ de umidade. Já para madeira de Araucaria, o referido manual atribui valores de 0,46 
$\mathrm{g} / \mathrm{cm}^{3}$ para a massa específica básica e $113000 \mathrm{kgf} / \mathrm{cm}^{2}$ para o MOE a $12 \%$ de umidade.

Pesquisas, conduzidas no Brasil, evidenciaram para Pinus sp de 20 anos e madeira de árvores maduras de Araucaria, respectivamente, massa específica de 0,42 e $0,58 \mathrm{~g} / \mathrm{cm}^{3}$ a $15 \%$ de umidade, e MOE de 60000 e $105000 \mathrm{kgf} / \mathrm{cm}^{2}$ (STUMPP, 1992). Analisando as propriedades da madeira de povoamentos com 18 anos de idade, TOMASELLI (1980) constatou que a massa específica e a resistência a flexão estática da Araucaria angustifolia foram maiores do que para $P$. elliottii e $P$. taeda.

DURLO (1988) verificou que a massa específica da madeira de desbaste de plantios de Pinus elliottii com 15 anos, determinada a $12 \%$ de umidade, variou entre 0,37 e $0,53 \mathrm{~g} / \mathrm{cm}^{3}$, ao passo que o MOE oscilou entre 56000 e $138000 \mathrm{kgf} / \mathrm{cm}^{2}$.

Os poucos estudos, conduzidos acerca da qualidade da madeira das florestas plantadas com incentivos fiscais, evidenciam resultados bastante variáveis entre regiões e uma larga amplitude de variação dos dados. Embora essas variações sejam comuns em pesquisas dessa natureza, as intervenções silviculturais e de manejo, bem como as diferenças edafo-climáticas regionais também contribuem para as desigualdades tecnológicas.

O presente trabalho teve como objetivo comparar entre sí, as propriedades físicas e mecânicas da madeira de Araucaria angustifolia, Pinus elliottii e Pinus taeda oriundas de florestas plantadas no sul do Brasil, bem como confrontá-las com informações disponíveis na literatura.

\section{MATERIAL E MÉTODOS}

O material, utilizado no presente estudo, foi coletado na Empresa Araupel S.A., localizada em Quedas do Iguaçu - PR. Árvores de Pinus elliottii e Pinus taeda obtidas de povoamentos individuais com 13 anos, submetidos a um desbaste entre o $8^{\circ}$ e $10^{\circ}$ ano, e de Araucária angustifolia com 19 anos, que já haviam sofrido dois desbastes, aos 10 e 15 anos, foram abatidas em talhões, previamente, selecionados.

Em cada um dos talhões sorteados, mediu-se o diâmetro à altura do peito (DAP) de todas as árvores, com base no qual se determinou a média aritmética (X) e o desvio padrão (DP). Com base nessas informações, identificaram-se as árvores de boa forma florestal que se encontravam no intervalo DAP $=\mathrm{X} \pm \mathrm{DP}$, e mais próximas dos pontos de amostragem, previamente, selecionados. Os valores de $\mathrm{X}$, encontrados para as duas espécies de Pinus e para a Araucaria, foram respectivamente, $23 \mathrm{~cm}$ e $24 \mathrm{~cm}$.

Para determinar a massa específica básica da madeira, foram abatidas cinco árvores de cada espécie estudada, retirando-se um disco de cada uma das seis posições predeterminadas: 0 (base), DAP, 25, 50, 75 e 100\% da altura comercial. O diâmetro mínimo, admitido para confecção do disco, foi de $8,0 \mathrm{~cm}$. Após sua confecção, os discos foram codificados e embalados em sacos plásticos, para minimizar a perda de umidade.

Para as demais propriedades, abateram-se mais cinco árvores de cada espécie as quais foram 
transformadas em toras de 2,50 m. Após o seccionamento, sorteou-se uma única tora de cada árvore, segundo a recomendação D 5536-94 da norma ASTM (1995), da qual retirou-se um pranchão central, de 8,0 cm de espessura com a medula incluída, e bem-orientado. O emprego da norma norte-americana, além de qualificar a madeira para o mercado dos Estados Unidos, permite a comparação com as espécies de Pinus que crescem na forma nativa naquele país.

Todo o material foi transportado para o Laboratório de Produtos Florestais do Departamento de Ciências Florestais da Universidade Federal de Santa Maria onde os discos foram mantidos submersos em água até a saturação, e os pranchões, gradeados em local arejado para uma secagem lenta e gradual.

Depois de secos, a espessura dos pranchões foi reduzida de 8,0 para $6,0 \mathrm{~cm}$ por meio de uma plaina desengrossadeira. Em seguida, foram transformados em peças de 6,0 x 6,0 cm, tendo-se o cuidado de eliminar a presença da medula.

Os procedimentos de confecção, condicionamento e ensaio dos corpos de prova seguiram a recomendação D 143-94 da norma ASTM (1995). Nos ensaios de flexão estática, empregou-se o método secundário para confecção dos corpos de prova, com dimensões de $2,5 \times 2,5 \times 41,0 \mathrm{~cm}$. O número de corpos de prova, bem como suas dimensões, velocidade do ensaio e demais informações para cada uma das propriedades analisadas são apresentadas na Tabela 1.

TABELA 1: Velocidade de ensaio, número e dimensões dos corpos de prova utilizados na determinação das propriedades físicas e mecânicas das três coníferas.

\begin{tabular}{|c|c|c|c|c|}
\hline \multirow{2}{*}{ PROPRIEDADE } & \multicolumn{3}{|c|}{ CORPO DE PROVA } & \multirow{2}{*}{$\begin{array}{c}\text { VELOCIDADE } \\
\text { DE ENSAIO } \\
(\mathrm{mm} / \mathrm{min})\end{array}$} \\
\hline & $\begin{array}{c}\text { N./ } \\
\text { ÁRVORE }\end{array}$ & $\begin{array}{c}\text { N./ } \\
\text { ESPÉCIE }\end{array}$ & $\begin{array}{c}\text { DIMENSÕES } \\
(\mathrm{cm})\end{array}$ & \\
\hline Massa específica básica & 6 & 30 & discos & - \\
\hline Coeficiente de contração & 3 & 15 & $5,0 \times 5,0 \times 15,0$ & - \\
\hline Flexão estática & 4 & 20 & $2,5 \times 2,5 \times 41,0$ & 1,3 \\
\hline Compressão axial & 3 & 15 & $5,0 \times 5,0 \times 20,0$ & 0,6 \\
\hline Compressão normal às fibras & 3 & 15 & $5,0 \times 5,0 \times 15,0$ & 0,3 \\
\hline Cisalhamento R e T & 6 & 30 & $5,0 \times 5,0 \times 6,25$ & 0,6 \\
\hline Tração normal às fibras $\mathrm{R} \mathrm{e} \mathrm{T}$ & 6 & 30 & $5,0 \times 5,0 \times 6,25$ & 2,5 \\
\hline Fendilhamento & 6 & 30 & $5,0 \times 5,0 \times 9,52$ & 2,5 \\
\hline Dureza Janka A, R e T & 3 & 15 & $5,0 \times 5,0 \times 15,0$ & 6,0 \\
\hline
\end{tabular}

Em que: $\mathrm{A}=$ axial; $\mathrm{R}=$ radial; $\mathrm{T}=$ tangencial

Após sua confecção, os corpos de prova foram condicionados em câmara climática a uma temperatura de $20^{\circ} \mathrm{C}$ e $65 \%$ de umidade relativa. Nessas condições, foram mantidos até que não ocorressem mais variações em peso, ou seja, até a madeira atingir uma umidade de equilíbrio de, aproximadamente, $12 \%$.

Não se incluem, nessa situação, o material utilizado nos testes de massa específica básica o qual foi conservado submerso em água. Depois de saturados, determinou-se o volume dos corpos de 
prova pelo método B da norma ASTM 2395-93 (1995). Após, eles foram submetidos à secagem até massa constante, como indicado no método D 4442-92 da mesma norma, calculando-se, então, a massa específica básica por meio da relação massa seca/volume saturado (ASTM, 1995).

Para a execução dos testes mecânicos, utilizou-se uma máquina universal de ensaio da marca Amsler, com capacidade para 20 toneladas, e dotada de acessórios específicos para cada teste. As leituras de carga e deformação foram obtidas por meio de um sistema informatizado de aquisição de dados, transferidas para uma planilha eletrônica e, posteriormente, procedeu-se à análise, usando-se pacotes estatísticos.

Por meio de um software estatístico, comparou-se as propriedades da madeira das três espécies estudadas mediante da análise da variância, desvio padrão, intervalo de confiança e probabilidade de erro.

\section{RESULTADOS E DISCUSSÃO}

A comparação entre as propriedades físicas e mecânicas da madeira de Pinus elliottii, Pinus taeda e Araucaria angustifolia, bem como a análise estatística dos dados são apresentadas na Tabela 2. Além do valor médio calculado para cada espécie, são mostrados também para cada propriedade, o intervalo de confiança, desvio padrão, coeficiente de variação, e probabilidade de erro. Para comprovar a existência de diferença ou igualdade estatística entre as médias, utilizou-se o teste LSD (least significance difference) ao nível de 5\% de probabilidade de erro.

A massa específica básica não mostrou diferença significativa entre as três espécies estudadas, confirmando estudos anteriores (TOMASELLI, 1980), sendo que as médias oscilaram entre 0,40 e $0,41 \mathrm{~g} / \mathrm{cm}^{3}$. Os baixos valores de desvio padrão e coeficiente de variação evidenciam a pequena variabilidade dos dados, e foram menores que aqueles apresentados na literatura (WOOD HANDBOOK, 1987; IBAMA, 1993).

Embora os coeficientes de contração radial, tangencial e volumétrica, encontrados para a madeira de Pinus elliottii, tenham sido menores que aqueles observados para as outras duas espécies, nenhuma diferença significativa entre eles foi detectada. Entretanto, a comparação das médias mostrou coeficientes de variação, relativamente, altos o que evidencia uma variabilidade, razoavelmente, grande entre os valores de contração determinados.

Em flexão, não foi detectada diferença significa entre os MOR das três coníferas. Porém, o MOE e tensão, no limite de proporcionalidade $\left(\sigma_{\mathrm{LP}}\right)$ da madeira de Araucaria, foram significativamente superiores àqueles encontrados para as espécies de Pinus. A comparação das médias pelo teste LSD evidencia ainda que, em $P$. elliottii e $P$. taeda, essas propriedades não diferem entre si.

Uma comparação, entre os valores de flexão estática citados na literatura (WOOD HANDBOOK, 1987) e os observados no presente estudo, é ilustrada na Figura 1. Observa-se que a diferença entre ambos, tanto para o MOR como para o MOE, é menor para a madeira de Araucaria. 
TABELA 2: Quadro comparativo das propriedades físicas e mecânicas da madeira de três coníferas provenientes de florestas plantadas.

\begin{tabular}{|c|c|c|c|c|c|c|}
\hline \multirow[t]{2}{*}{ PROPRIEDADE } & \multicolumn{3}{|c|}{ VALOR MÉDIO $^{1} \pm$ ERRO } & \multirow[t]{2}{*}{$S$} & \multirow{2}{*}{$\begin{array}{l}\mathrm{CV} \\
\% \\
\end{array}$} & \multirow[t]{2}{*}{$\mathrm{P}$} \\
\hline & P. elliottii & P. taeda & A. angustifolia & & & \\
\hline Massa específica básica & $0,41 \pm 0,02 \mathrm{a}$ & $0,41 \pm 0,02 \mathrm{a}$ & $0,40 \pm 0,02 \mathrm{a}$ & 0,04 & 3,2 & 0,4079 \\
\hline $\begin{array}{l}\text { Coeficiente de contração: } \\
\text { radial } \\
\text { tangencial } \\
\text { volumétrica }\end{array}$ & $\begin{array}{l}3,9 \pm 0,6 \mathrm{a} \\
5,8 \pm 0,6 \mathrm{a} \\
9,8 \pm 0,9 \mathrm{a} \\
\end{array}$ & $\begin{array}{r}4,4 \pm 0,6 \mathrm{a} \\
6,5 \pm 0,6 \mathrm{a} \\
10,9 \pm 0,8 \mathrm{a} \\
\end{array}$ & $\begin{array}{r}4,4 \pm 0,6 \mathrm{a} \\
6,1 \pm 0,6 \mathrm{a} \\
10,4 \pm 0,9 \mathrm{a} \\
\end{array}$ & $\begin{array}{l}1,1 \\
1,2 \\
1,5 \\
\end{array}$ & $\begin{array}{l}26,0 \\
19,6 \\
14,5 \\
\end{array}$ & $\begin{array}{l}0,3844 \\
0,2550 \\
0,1950 \\
\end{array}$ \\
\hline $\begin{array}{l}\text { Flexão estática: } \\
\text { módulo de ruptura } \\
\text { módulo de elasticidade } \\
\sigma_{\mathrm{LP}}\end{array}$ & $\begin{aligned} & 555 \pm 43 \mathrm{a} \\
& 73266 \pm 7898 \mathrm{~b} \\
& 170 \pm 14 \mathrm{~b} \\
&\end{aligned}$ & $\begin{aligned} & 556 \pm 46 a \\
& 59561 \pm 8349 b \\
& 152 \pm 16 b \\
&\end{aligned}$ & $\begin{aligned} & 643 \pm 43 a \\
& 97636 \pm 7898 \mathrm{a} \\
& 218 \pm 15 \mathrm{a} \\
&\end{aligned}$ & $\begin{array}{c}132 \\
24262 \\
44 \\
\end{array}$ & $\begin{array}{l}22,6 \\
31,6 \\
24,4 \\
\end{array}$ & $\begin{array}{l}0,0700 \\
0,0001 \\
0,0001 \\
\end{array}$ \\
\hline $\begin{array}{l}\text { Compressão axial: } \\
\text { módulo de ruptura } \\
\text { módulo de elasticidade } \\
\sigma_{\mathrm{LP}}\end{array}$ & $\begin{aligned} 292 & \pm 22 b \\
73531 & \pm 9533 b \\
196 & \pm 25 b\end{aligned}$ & $\begin{array}{c}291 \pm 24 b \\
87606 \pm 10443 b \\
186 \pm 28 b\end{array}$ & $\begin{array}{c}373 \pm 21 \mathrm{a} \\
119778 \pm 9159 \mathrm{a} \\
260 \pm 25 \mathrm{a}\end{array}$ & $\begin{array}{c}51,6 \\
22928 \\
61,7\end{array}$ & $\begin{array}{l}23,5 \\
24,5 \\
28,7\end{array}$ & $\begin{array}{l}0,0004 \\
0,0001 \\
0,0118\end{array}$ \\
\hline $\begin{array}{l}\text { Compressão normal: } \\
\text { módulo de elasticidade } \\
\sigma_{\mathrm{LP}}\end{array}$ & $\begin{aligned} 2595 & \pm 582 \mathrm{a} \\
54 & \pm 8 \mathrm{a}\end{aligned}$ & $\begin{aligned} 2960 & \pm 555 a \\
50 & \pm 6 a\end{aligned}$ & $\begin{array}{c}3334 \pm 555 a \\
58 \pm 10 a\end{array}$ & $\begin{array}{l}900 \\
13,0\end{array}$ & $\begin{array}{l}30,4 \\
23,2\end{array}$ & $\begin{array}{l}0,1885 \\
0,8242\end{array}$ \\
\hline Cisalhamento & $85 \pm 4 a$ & $87 \pm 4 a$ & $91 \pm 3 a$ & 14,0 & 16,0 & 0,2476 \\
\hline Tração normal às fibras & $18 \pm 2 b$ & $24 \pm 2 \mathrm{a}$ & $18 \pm 2 b$ & 7,0 & 35,0 & 0,0020 \\
\hline Fendilhamento & $4,5 \pm 0,3 \mathrm{a}$ & $4,8 \pm 0,3 \mathrm{a}$ & $4,8 \pm 0,3 \mathrm{a}$ & 1,0 & 21,3 & 0,4144 \\
\hline $\begin{array}{l}\text { Dureza Janka: } \\
\text { radial } \\
\text { tangencial } \\
\text { axial }\end{array}$ & $\begin{array}{l}224 \pm 31 a \\
293 \pm 43 a \\
336 \pm 35 b\end{array}$ & $\begin{array}{l}222 \pm 31 a \\
302 \pm 43 a \\
315 \pm 35 b\end{array}$ & $\begin{array}{l}236 \pm 33 a \\
252 \pm 45 a \\
370 \pm 36 a\end{array}$ & $\begin{array}{l}52 \\
73 \\
60\end{array}$ & $\begin{array}{l}22,9 \\
25,9 \\
17,6\end{array}$ & $\begin{array}{l}0,7978 \\
0,2376 \\
0,0987\end{array}$ \\
\hline
\end{tabular}

Em que: ${ }^{1}$ Densidade básica em g/ $\mathrm{cm}^{3}$; coeficiente de contração em $\%$; propriedades mecânicas em $\mathrm{kgf} / \mathrm{cm}^{2} ; \sigma_{\mathrm{LP}}=$ tensão no limite de proporcionalidade; $\mathrm{s}=$ desvio padrão nas unidades especificadas; $\mathrm{CV}=$ coeficiente de variação, em $\% ; \mathrm{P}=$ probabilidade de erro; Em cada linha, médias seguidas da mesma letra não diferem entre si pelo teste LSD (P > $0,05)$.

Em compressão axial às fibras, tanto o MOR e o MOE como a $\sigma_{\mathrm{LP}}$ foram significativamente maiores para madeira de Araucaria do que para as de $P$. elliottii e $P$. taeda, sendo que os valores das duas últimas não diferiram entre si em níveis de probabilidade de erro inferiores a $1 \%$. Por outro lado, em compressão normal às fibras, foi detectada uma igualdade estatística entre as três espécies de coníferas, tanto para o MOE como para a $\sigma_{\mathrm{LP}}$. Entretanto, a elevada probabilidade de erro (82\%), verificada para a $\sigma_{\mathrm{LP}}$, sugere a possibilidade da diferença ser em virtude do acaso.

Apesar dos valores não diferirem estatisticamente, a Araucaria apresentou uma resistência média ao cisalhamento $\left(91 \mathrm{kgf} / \mathrm{cm}^{2}\right)$, ligeiramente, superior às madeiras de Pinus. Já com relação ao fendilhamento, a resistência foi praticamente a mesma nas três espécies. 

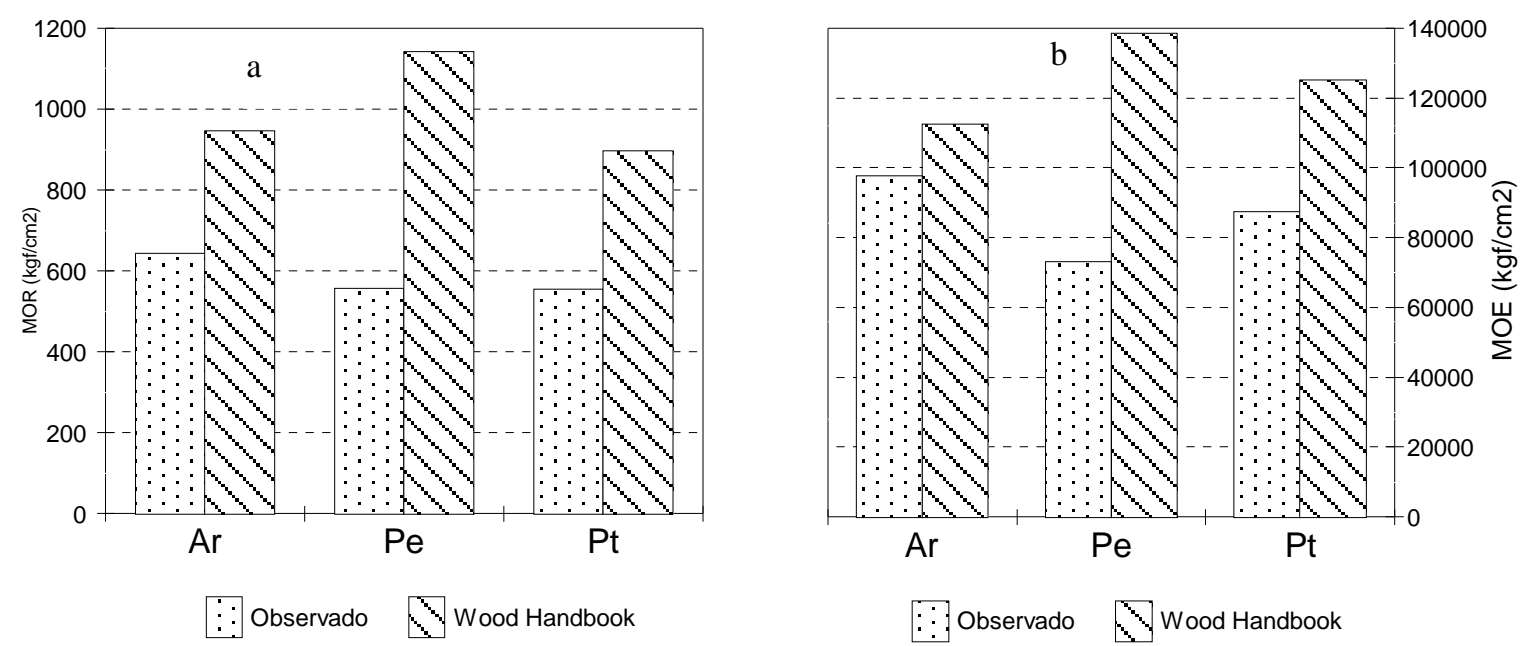

FIGURA 1: Comparação entre os valores de MOR (a) e de MOE (b), observados e de literatura, para as madeiras de Araucaria (Ar), P. elliottii (Pe) e P. taeda (Pt).

A madeira de $P$. taeda foi a mais resistente em tração normal às fibras $\left(24 \mathrm{kgf} / \mathrm{cm}^{2}\right)$, enquanto que tanto o $P$. elliottii como a Araucaria apresentaram o mesmo valor de $18 \mathrm{kgf} / \mathrm{cm}^{2}$, a uma probabilidade de erro de $0,2 \%$. A variabilidade evidenciada, pelo alto coeficiente de variação (35\%), deve-se à dispersão nos dados resultantes do uso de corpos de prova orientados nos dois sentidos, uma vez que, tanto a área de contato entre os raios e as células axiais como a transição lenho tardiolenho inicial, possuem baixa resistência à tração perpendicular.

A dureza, nos sentidos radial e tangencial, foi igual entre as três espécies estudadas, porém no sentido axial, a resistência média de $370 \mathrm{kgf} / \mathrm{cm}^{2}$, encontrada para a madeira de Araucaria, foi significativamente maior que as demais. Nenhuma diferença foi observada entre os valores 336 e 315 $\mathrm{kgf} / \mathrm{cm}^{2}$ obtidos, respectivamente, para as madeiras de $P$. elliottii e $P$. taeda nesse mesmo sentido.

Os valores médios, observados no presente estudo, para flexão estática e massa específica, foram inferiores àqueles citados na literatura para madeiras provenientes de florestas nativas de Pinus (USA) e Araucaria o que pode ser atribuído aos fatores de sítio e à idade do povoamento. A madeira de Araucaria foi a que apresentou valores mais próximos daqueles encontrados em madeira adulta da mesma espécie. Árvores originárias de florestas maduras são formadas quase que, na sua totalidade, de madeira adulta, possuindo maior resistência mecânica que a obtida de povoamentos jovens. A presença de células, relativamente, mais longas e de paredes mais espessas, bem como a maior proporção de lenho tardio, conferem à madeira adulta maior massa específica e, consequentemente, propriedades de resistência mais elevadas. 


\section{CONCLUSÕES}

A análise comparativa da madeira, das três coníferas provenientes de florestas plantadas, evidenciou que nenhuma diferença significante foi observada na massa específica básica e no coeficiente de contração. De um modo geral, os valores médios das propriedades mecânicas da Araucaria angustifolia foram numericamente maiores que as duas espécies de Pinus, porém diferenças significativas foram encontradas apenas em flexão estática (MOE e $\sigma_{\mathrm{LP}}$ ), em compressão axial e dureza axial. Os valores de resistência mecânica foram iguais entre as duas espécies de Pinus, com exceção do teste de tração normal às fibras onde o valor encontrado para $P$. taeda foi estatisticamente diferente, ao nível de 5\% de probabilidade, do P. elliottii e da Araucaria.

Recomenda-se a condução de novos estudos com material de árvores maduras, para comparar a qualidade da madeira adulta entre as espécies. Adicionalmente, sugere-se estabelecer correlações entre as propriedades da madeira e as características de crescimento, tais como lenho juvenil, percentagem de lenho tardio e largura do anel de crescimento.

\section{AGRADECIMENTO}

Os autores agradecem à empresa Araupel S.A., por possibilitar a realização deste trabalho.

\section{REFERÊNCIAS BIBLIOGRÁFICAS}

ASTM. American Society for Testing and Materials. Standard methods of testing small clear specimens of timber, D 143-94. Philadelphia, 1995a.

Test methods for specific gravity of wood and wood-base materials., D 2395-93. Philadelphia, 1995b.

Test methods for direct moisture content measurement of wood and wood-base materials., D 4442-92. Philadelphia, 1995c.

. Practice for sampling forest trees for determination of clear wood properties., D 5535-94. Philadelphia, 1995d.

BIBLIS, E.J.; BRINKER, R.; CARINO, H.F. et al. Effect of stand age on flexural properties and grade compliance of lumber from loblolly pine plantation timber. For. Prod. J., Madison, v. 43, n.2, p. 23-28, feb. 1993.

DURLO, M.A. Determinação de variáveis para caracterização de Pinus elliottii com finalidade estrutural. In: CONGRESSO FLORESTAL ESTADUAL 6., 1988. Nova Prata, RS. Anais ... Nova Prata, 1988. p. 1097 - 1113.

IBAMA. Instituto Brasileiro do Meio Ambiente e dos Recursos Naturais Renováveis. Amostragem e propriedades físico-mecânicas de madeiras amazônicas. Brasília, 1993. 103p. 
KELLISON, R.C. Characteristics affecting quality of timber from plantations, their determination and scope for modification. In: IUFRO WORLD CONGRESS 17., 1981, Kyoto. Proceedings ... Kyoto, 1981. p. 77-87.

LADRACH, W.E. Control of wood properties in plantations. In: IUFRO WORLD CONGRESS 18., 1986, Ljubljana. Proceedings ... Ljubljana, 1986. p. 369-379.

McALISTER, R.H.; CLARK III, A. Effect of geographic location and seed source on the bending properties of juvenile and mature loblolly pine. For. Prod. J., Madison, v. 41, n. 9, p. 39-42, Sept. 1991.

PEARSON, R.G.; GILMORE, R.C. Characterization of the strength of juvenile wood of loblolly pine (Pinus taeda). For. Prod. J., Madison, v. 21, n. 1, p. 23-30, May 1971.

. Effect of fast growth rate on the mechanical properties of loblolly pine. For. Prod. J., Madison, v. 30, n. 5, p. 47-54, May 1980.

STUMPP, E. Valores físicos-mecânicos de Pinus spp. em comparação com Araucaria angustifolia. In.: CONGRESSO FLORESTAL ESTADUAL 7., 1992, Nova Prata, RS. Anais ... Nova Prata, 1992. p. 1413-1420.

TOMASELLI, I. Comparação da qualidade da madeira de Araucaria angustifolia e Pinus spp. produzida em reflorestamentos. In.: ENCONTRO DA IUFRO SOBRE PROBLEMAS FLORESTAIS DO GÊNERO ARAUCARIA, 1979, Curitiba. Anais ... Curitiba, 1980. p. 286291.

WOOD HANDBOOK: Wood as an engineering material. Washington, DC. US. Department of Agriculture, Forest Products Laboratory, 1987. (revisado). (Agriculture Handbook, 72). 